\title{
Markt, Hierarchie und die dazwischen liegende Vielfalt: hybride Organisationen und Selbstverwal- tungen von Allmenderessourcen regen zu innovativen Kooperationen an
}

Corporate Governance; Gemeinschaftsgüter; hybride Organisationen; Institutionenökonomik; Netzwerke; Neue Kooperationen

Die mit dem Wirtschaftsnobelpreis 2009 ausgezeichneten Wissenschaftler Elinor Ostrom und Oliver E. Williamson widmen sich beide Fragestellungen der Institutionenökonomik. Der Beitrag informiert über beide Nobelpreisträger und ihre Ansätze.

\section{Einleitung}

Gemeinsam mit Oliver E. Williamson wurde Elinor Ostrom 2009 mit dem Wirtschaftsnobelpreis für deren ,analysis of economic governance“ (Nobelprize.org 2009) ausgezeichnet, wobei Elinor Ostrom „especially the commons“" ${ }^{\text {"1 }}$ und Oliver E. Williamson „especially the boundaries of the firm“'2 wissenschaftlich erfolgreich untersuchten. Beide

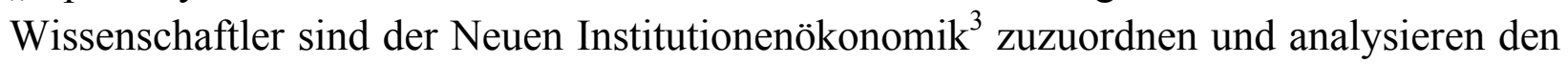
Einfluss unterschiedlicher Institutionen ${ }^{4}$ auf die Lösung von Koordinations- und Anreizproblemen wirtschaftlichen Handelns. Dabei verbleiben die Preisträger nicht in modelltheoretischen Betrachtungen, sondern testen die empirische Relevanz ihrer Modelle und konfrontieren sie mit den Gegebenheiten der Wirtschaftspraxis. Prononciert sagt dazu

1 Aus der Fülle ihrer Schriften sei auf Elinor Ostrom (1990) hingewiesen, denn diese Monographie erschien auch auf deutsch (Ostrom 1999).

2 Ebenfalls aus zahlreichen Publikationen sei hier hingewiesen auf: Oliver E. Williamson (1985); auch diese Monographie erschien auf deutsch (Williamson 1990). Zu seinen bekanntesten Arbeiten zählt auch sein Werk Markets and Hierarchies: Analysis and Antitrust Implications (Williamson 1975).

3 Der Begriff „Neue Institutionenökonomik“ wurde von Williamson erstmals 1975 eingeführt. Auf S. 1 heißt es: "A broad based interest among economists in what might be referred to as the ,new institutions economics" has developed in recent years" (Williamson 1975); vgl. auch Groth 2009, S. 771.

4 Der Begriff der Institution ist ein Grenzbegriff zwischen Soziologie und Ökonomie. Viele Soziologen setzten bei der Etablierung der wissenschaftlichen Disziplin „Soziologie“ ihr Fach mit der Analyse von Institutionen und institutionellem Verhalten gleich. Die Neue Institutionenökonomik definiert den Begriff der Institution als ein ,,auf ein bestimmtes Zielbündel abgestelltes System von Normen einschließlich deren Garantieinstrumente (darunter Verfügungsrechte und ihre Garantien). Sie [die Institution] steuert, wenn auch natürlich nicht perfekt, das soziale Handeln der Individuen.“ (Richter 1990, S. 572; siehe auch: Zerche/Schmale/Blome-Drees 1998, S. 57 f.) 
Elinor Ostrom: „Es ist meine Überzeugung, dass Wissen sich durch das kontinuierliche Hin- und Herpendeln zwischen empirischer Beobachtung und intensiver theoretischer Arbeit akkumuliert“ (Ostrom 1999, S. XVI). Die Veröffentlichungen beider Preisträger zeigen, dass sie nach dieser Überzeugung erfolgreich handeln.

\section{Neue Institutionenökonomik und Oliver E. Williamson}

Unzweifelhaft ist die ,kurze Geschichte der Neuen Institutionenökonomik [...] eine Erfolgsgeschichte“ (Voigt 2002, S. 15). Ihre erkenntnisleitende Fragestellung nach der Bedeutung von Institutionen für die Entwicklung der Wirtschaft sowie für die Bewältigung von Koordinations- und Anreizstrukturen des wirtschaftlichen Verhaltens hat in den vergangenen Jahrzehnten viele Vertreter der Wirtschaftswissenschaften angezogen. Dabei ist eine ökonomische Institutionenlehre keineswegs neu, sondern war von Anfang an bei der Entwicklung der wissenschaftlichen Disziplin der damals sog. Nationalökonomie dabei (Schellschmidt 1997). Ebenso wie der Keynesianismus interessierte sich die über Jahrzehnte dominierende Neoklassische Theoriebildung nicht für das wirtschaftliche Handeln der einzelnen Wirtschaftssubjekte, das durch formelle und informelle Regeln und Normen - eben durch Institutionen - geprägt ist, sondern beförderte dieses in die Annahmen, die den Ansätzen zugrunde liegen und verdrängten so die älteren Ansätze. So wurde auch die Nutzung des Marktes, die Herstellung von Transparenz und die damit verbundenen Informations- und Suchkosten sowie der Abschluss von Verträgen mit Überwachungskosten der Einhaltung aus der Analyse der Marktprozesse ausgeblendet, weshalb diese sog. Transaktionskosten lange Jahre unberücksichtigt blieben.

Schon seit einiger Zeit sind wichtige Vordenker wie etwa James M. Buchanan (im Jahr 1986), Begründer der Forschungsrichtung wie Ronald R. Coase (im Jahr 1990) und Weiterentwickler wie Douglass North (im Jahr 1993) mit dem Nobelpreis ausgezeichnet worden und die (Neue) Institutionenökonomik gehört heute mit zum Standard der Lehre und Forschung.

Die Entdeckung von Coase als er in den 1930er Jahren die Frage stellte: „Warum gibt es überhaupt Unternehmen?“(Coase 1937), dass die mit der Marktnutzung einhergehenden Transaktionskosten dazu führen, dass nicht allein der Markt wirtschaftlichen Austausch koordiniert, sondern Hierarchien wie Unternehmen (oder der Staat) dies unter Umständen kostengünstiger leisten, fand lange Zeit keinen Widerhall in der Wissenschaft. Erst in den 1960er Jahren wurde zunehmend die Frage nach Eigentums- bzw. Verfügungsrechten bzw. nach den sozialen Kosten aufgrund externer Effekte seitens der Finanzwissenschaftler in den sog. Public Choice Theorien gestellt.

Williamson selber nennt in seiner Rede anlässlich der Preisverleihung (Williamson 2009; Groth 2009, S. 770-776) seine Mitstreiter bei der Ausformulierung der Neuen Institutionenökonomik. Auf John R. Commons (1932) beruft er sich, wenn die Transaktion die zentrale Kategorie des Ansatzes wird. Von James Buchanan (1975) übernimmt er die (Public) Choice-Perspektive sowie den Contract-Approach und bezieht darauf die Trans- 
aktionskosten-Ökonomie, um die unterschiedlichen Transaktionen und die verschiedenen Governance-Strukturen zu analysieren. Herbert Simon (bounded rationality), James March und Richard Cyert und nicht zuletzt Kenneth Arrow (1969) beeinflussten ihn mit ihrer Sichtweise der Organisationstheorie. Zu nennen ist auch Chester Barnard (1938) und dessen Argumente ,(1) that adaptation is the central problem of economic organization and (2) that cooperative adaptations within firms that are accomplished under the auspices of hierarchy"(Williamson 2005, S. 5). Friedrich August von Hayek, der in Wien Rechtswissenschaften, Nationalökonomie sowie Psychologie studierte, beeinflusste ihn mit seiner liberalen Sichtweise, wobei Williamson anders als von Hayek nicht nur auf die Anpassung autonomer ökonomischer Akteure setzt, die auf Preissignale des Marktes spontan reagieren, sondern neben der „autonomen Anpassung“ die „koordinierte Anpassung“ in Hierarchien betrachtet (Williamson 2005, S. 5ff.). Nicht zuletzt ist auch Ronald Coase zu nennen mit seinem Aufsatz „The Nature of the Firm“ und in dem 1960 von ihm behandelten Problem der Sozialen Kosten, das die Frage nach den Externalitäten beinhaltet. Das Problem, warum externe Effekte nicht in Einzelverträgen (hinreichend) erfasst werden und die Frage, welche Einflüsse dieses Vertragsversagen auf die Effizienz der Allokation haben kann (Kliemt 2009a, S. 644), werden von Williamson ausführlich analysiert.

Williamson wendet sich der Analyse der „vertikalen Integration der Produktion“ zu und damit der Frage, was eigentlich das bis dahin von der Volkswirtschaftlehre als „black box" behandelte Unternehmen ausmacht. Ihn interessiert weniger die Produktionsseite in einem Unternehmen, sondern er betrachtet die Governance-Strukturen und analysiert die in einem Unternehmen zu schließenden Verträge. Als transaktionskostenrelevante Einflussgrößen identifiziert Williamson Spezifität (z. B. ist die Nachfrage nach Schraubengröße, die nicht DIN Norm erfüllt, von hoher Spezifität), Unsicherheit (z. B. kann von dem Risiko aufgrund der Währungsschwankungen bei einem Auslandsgeschäft große Unsicherheit ausgehen), Komplexität (der Bau einer Großanlage durch viele kleine Spezialfirmen kann ein sehr komplexes Projekt sein), und Häufigkeit einer Austauschbeziehung. Dabei spielt natürlich auch das menschliche Verhalten eine Rolle, von dem angenommen wird, dass es sich durch begrenzte Rationalität sowie strategisches Verhalten bzw. Opportunismus auszeichnet. Diese Annahmen spielen vor allem bei der Gestaltung von Verträgen eine wichtige Rolle, denn diese müssen alle potenziell möglichen Fälle des Verhaltens der Vertragspartner und deren Konsequenzen berücksichtigen.

Williamson bezieht sein Analysegerüst auf die Frage, warum Unternehmen andere übernehmen und warum andere Unternehmen Teile der Produktion auslagern und damit die vertikale Integration der Produktion zugunsten von Marktbeziehungen abbauen. Auch betrachtet er die sog. hybriden Organisationen und analysiert unterschiedliche Organisationen wie Unternehmensnetzwerke, strategische Allianzen, Genossenschaften und Public-Private-Partnerships.

Nach Groth beruht die Sonderstellung Williamsons innerhalb der Wissenschaftler, deren Forschungen im Rahmen der Neuen Institutionenökonomik anzusiedeln sind, ,maßgeblich in der umfassenden Weiterentwicklung des Analyserahmens der Transaktionskosten 
unter Berücksichtigung der Relevanz sozialer Komponenten und den in der Praxis relevanten Ausprägungen menschlichen Verhaltens. Dabei sind - neben der Einbeziehung von begrenzter Rationalität und Opportunismus - insbesondere der Einfluss der Faktorspezifitäten, der schließlich in das Konzept der Fundamentalen Transformation mündet, sowie die Ableitung grundlegender institutioneller Arrangements zur Ausgestaltung von Transaktionsbeziehungen hervorzuheben. Im Zuge dessen konnte Williamson umfassend die Relevanz von Transaktionskosten aufzeigen"(Groth 2009, S. 775). Dabei macht Williamson aber auch darauf aufmerksam, dass ,complex phenomena (networks included) are usefully examined from several perspectives, of which TCE [Transaction Cost Theory; d.V.] is merely one. I would nevertheless advise that all would-be theories of networks be guided by the same four precepts of pragmatic methodology: keep it simple, make it plausible, and drive refutable implication to which the data are thereafter applied" (Williamson 2005, S. 25).

\section{Die besondere Bedeutung der institutionellen Diversität und Elinor Ostrom}

Auch Elinor Ostrom kann auf ein erfülltes Wissenschaftlerleben unter dem Zeichen der Institutionenökonomik zurückblicken. Während Williamson stärker zur ökonomischen Theorie des Rechts tendiert, ist Ostrom eher dem Kontext von Public Choice (Neue Politische Ökonomie) zuzuordnen (Kliemt 2009a, S. 644). Kliemt sieht sie als im klassischen Sinne politische Ökonomin (Kliemt 2009). Anfang der 1960er Jahre lernte sie in einem Graduiertenkolleg unter der Leitung des inzwischen ebenfalls sehr renommierten Politikwissenschaftlers Vincent Ostrom, ihrem späteren Mann und engsten Kollegen, ${ }^{5}$ das Problem der gemeinsamen Nutzung von Wasserressourcen in Südkalifornien kennen. Eine über 40-jährige wissenschaftliche Auseinandersetzung mit Problemen des kollektiven Handelns von Allmenderessourcen nutzenden Individuen führt dazu, dass sie dem zwischen Markt und Staat liegenden intermediären Bereich als Forschungsgegenstand ihre Aufmerksamkeit widmet. Hier agieren die vielen sozialen Gruppen, die selbstorganisiert und selbstbestimmt Gemeinschaftsgüter verwalten, bereitstellen und sich aneignen.

Allmenderessourcen sind natürliche oder von Menschen geschaffene Gemeinschaftsgüter, von deren Nutzung potenzielle Aneigner nicht (oder kaum, da dies zu teuer ist) ausgeschlossen werden können. Ostrom unterscheidet zwischen einem Ressourcensystem (als Vorratsvariable wie z. B. Fischgründe, Weideland, Bewässerungskanäle etc.) und Ressourceneinheiten, die dem Ressourcensystem entnommen werden (als Flussvariable sind hier gefangene Fische, von Weidetieren verzehrtes Weidefutter, entnommene $\mathrm{Ku}$ bikmeter Wasser zu nennen). Während das Ressourcensystem gemeinsam genutzt werden kann, besteht bei den Ressourceneinheiten Rivalität im Konsum: Fische, die der eine

5 Gemeinsam mit ihrem Ehemann Vincent Ostrom gründete sie 1973 den „Workshop in Political Theory and Policy Analysis“ an der Indiana Universität, wobei der Begriff „Workshop“ dieser Institution vielleicht nicht gerecht wird. 
Fischer fängt, können nicht mehr von anderen gefangen werden - oder allgemeiner: jeder Aneigner der Allmenderessource vermindert mit seinem Konsum die aktuellen Konsummöglichkeiten weiterer Nutzer (Ostrom 1999, S. 38ff.). Bei den erneuerbaren Ressourcen wird bei angemessener Ressourcenentnahme das Ressourcensystem als solches erhalten. Schon Aristoteles erkannte in der Antike die „Tragik der Allmende“ (Hardin 1968), nämlich dass „dem Gut, das der größten Zahl gemeinsam ist, die geringste Fürsorge zuteil wird. Jeder denkt hauptsächlich an sein eigenes, fast nie an das gemeinsame Interesse.“6 Wirtschaftswissenschaftler und Politiker ziehen hier häufig nur zwei Optionen in Betracht: die Verfügungsrechte der Allmenderessourcen müssen entweder privatisiert oder unter staatliche Aufsicht gestellt werden. Elinor Ostrom gelingt es aber zu zeigen, dass lokale, genossenschaftliche oder genossenschaftsartige Selbsthilfe- und Selbstverwaltungsformen der Allmendenutzer Lösungsmöglichkeiten kreieren können, die Modellen der Übertragung ins Privateigentum oder der staatlichen Verwaltung überlegen sind.

Auch sie zieht für die Analyse ihres Ausgangsproblems die in den Wirtschaftswissenschaften bestehenden Ansätze heran (Ostrom 1999). Das Problem der Bereitstellung und Bewirtschaftung von Allmenderessourcen betrachtet sie so zunächst im Lichte der von Mancur Olson (1998) entwickelten Logik des kollektiven Handelns, welche bei jedem Nutzer solche Anreize ausmacht, die dazu führen, dass nur die privaten, nicht aber die kollektiven Kosten ins Kalkül einbezogen werden. Mit Hilfe des institutionenökonomischen Ansatzes formuliert sie erneut ihr Ausgangsproblem und betrachtet Lösungshinweise der Unternehmens- sowie der Staatstheorie. Sie kristallisiert die drei sie interessierenden Probleme heraus - die Bereitstellung neuer Institutionen, die Selbstverpflichtung sowie die Überwachung zur nachhaltigen Bereitstellung eines Allmenderessourcensystems mit der Möglichkeit der Aneignung von Ressourceneinheiten - und analysiert sie gesondert in Konfrontation mit den in der Literatur vorhandenen Modellen. Sie konkretisiert erneut ihre Fragestellung und definiert für ihre Zwecke die zentralen Begriffe.

Wiederholte Gastaufenthalte am Zentrum für interdisziplinäre Forschung an der Universität Bielefeld, ${ }^{7}$ insbesondere in der „Forschungsgruppe über Spieltheorie und Verhaltenswissenschaft", einer Gruppe um den einzigen Wirtschaftsnobelpreisträger aus Deutschland, Reinhard Selten, machten Elinor Ostrom mit den spieltheoretischen Grundlagen der Analyse kollektiver bzw. individueller Strategien bei Allmendeproblemen vertraut.

Das in der Theorie gefundene Rüstzeug konfrontiert sie konsequent mit Befunden aus der Praxis. Ob historische oder aktuelle Fallstudien, Laborexperimente mit spieltheoretischer Anordnung (Ostrom/Gardener/Walker 1994): weltweit findet Elinor Ostrom ihr empirisches Material, das sie gewissenhaft und penibel (Engelhardt 2001, S. 46) auf seine Erklärungskraft überprüft, wie es zu selbstorganisierten, genossenschaft(sähn)lichen Organisationen rund um Allmenderessourcen kommt und vor allem, welche Faktoren zum

6 Aristoteles, Politika, Buch II, Kap. 3, zitiert in: Ostrom (1999, S.3).

7 1981/1982 gehörte sie der Forschungsgruppe "Steuerung und Erfolgskontrolle im öffentlichen Sektor" unter Leitung des Bielefelder Soziologen Franz-Xaver Kaufmann und 1987/1988 der Forschergruppe um Reinhard Selten "Game Theory in the Behavioral Sciences" an. Vgl. Universität Bielefeld (2010). 
Erfolg und welche zum Misserfolg beitragen. Ihr geht es letztendlich nicht nur um eine Erklärung von Kooperationen, sondern um die Möglichkeit, Vorhersagen zu treffen. Dazu entwirft sie das „Institutional Analysis and Development (IAD) framework“, das weitere Forschungen kanalisiert und dem „Understanding Institutional Diversity“ (Ostrom 2005) hilft. Sie zeigt, ,how a theory of boundedly rational, norm-based human behaviour is a better foundation for explaining collective action than a model of maximizing material payoffs to self" (Ostrom 2009, S. 51) Sie arbeitet an einer "More General Theory of Human Behavior" (Ostrom 2009, S. 57) und betrachtet die Menschen in ihrer Anpassungsfähigkeit. „Human learn norms, heuristics, and full analytical strategies from one another, from feedback from the world, and from their own capacity to engage in selfreflection and imagine a differently structured world. They are capable of designing new tools - including institutions - that can change the structure of the worlds they face for good or evil purposes. ${ }^{8}$ They adopt both short-term and long-term perspectives dependent on the structure of opportunities they face. Multiple models are consistent with a theory of boundedly rational human behavior, including a model of complete rationality when paired with repetitive, highly competitive situations" (Ostrom 2009, S. 58).

Diese Lernfähigkeit des Menschen macht in den Augen Elinor Ostroms die Vielfalt der Institutionen so wertvoll: „Alles, was die Vielfalt fördert, schafft Sozialkapital und dient so der Gesellschaft. Zentrale Regelsysteme und Einheitslösungen sind nie hilfreich. Das ist mir wirklich wichtig: Die Vielfalt der gesellschaftlichen Optionen ist von überragender Bedeutung“ (Ostrom 2007, S. 17).

\section{Das Nobelpreiskomitee ehrt bekennend interdisziplinär ausge- richtete Forscherpersönlichkeiten}

Es ist bemerkenswert, dass beide Wirtschaftsnobelpreisträger nicht als reine Ökonomen ausgewiesen sind, sondern beide bewusst die Fachgrenzen überschreiten. Elinor Ostrom ist ausgewiesene Politikwissenschaftlerin und lehrt an der Indiana University, Bloomington, USA. Als Arthur F. Bentley Professor of Political Science ist sie zugleich Senior Research Director des von ihr und ihrem Mann, dem renommierten Politikwissenschaftler Vincent Ostrom, eingerichteten Workshop in Political Theory and Policy Analysis, Indiana University, Bloomington, ferner Founding Director im Center for the Study of Institutional Diversity, Arizona State University, Tempe und Professor (parttime) an der School of Public and Environmental Affairs, Indiana University, Bloomington.

Oliver E. Williamson ist seit 1988 Edgar F. Kaiser Emeritus Professor für Betriebswirtschaftslehre, Volkswirtschaftslehre und Rechtswissenschaften an der University of California, nachdem er wichtige universitäre Stationen in den USA - MIT, Stanford University, Carnegie-Mellon University, University of Pennsylvania, Yale University - absol-

8 Auch Sozialkapital kann für gute und schlechte Zwecke genutzt werden: ein beliebtes Beispiel in diesem Zusammenhang ist die Mafia, die ein hohes Ausmaß an Sozialkapital, an Vernetzung und starkem Zusammenhalt ausgebildet hat, leider aber diese selbstkreierten Institutionen für gesellschaftsschädliche Zwecke einsetzt. 
viert hat. In seiner Nobelpreisrede nennt Williamson den ihn bestimmenden „'Carnegie Triple': be disciplined; be interdisciplinary; have an active mind“ (Williamson 2009, Folie 12).

Vermutlich können an den Lebensläufen beider Nobelpreisträger typische Karrieremuster männlicher und weiblicher Wissenschaftler nachgezeichnet werden. Oliver E. Williamson durchlief seine Karriere an verschiedenen renommierten Universitäten in den USA und ist seit über 50 Jahren mit seiner Ehefrau, mit der er fünf Kinder hat, verheiratet. Elinor Ostrom hat es nun als erste Frau geschafft, den „Männerzirkus der Ökonomie“ (Hickel 1994, S. 176) aufzubrechen. Dabei war ihr der Weg einer wissenschaftlichen Karriere alles andere als vorgezeichnet, ,sie verdankt ihn ihrer unbekümmerten Hartnäckigkeit" (Horn 2009). Denn nach ihrem Collegeabschluss arbeitet Elinor Ostrom zunächst zur Sicherung des Lebensunterhaltes für sie selbst und ihren studierenden ersten Ehemann in einem Büro, später in der Personalverwaltung der University of California, wo sie beginnt, neben ihrer Arbeit Vorlesungen in Politologie zu besuchen. Ihr Interesse an den Politikwissenschaften führt dazu, dass sie ihren Job aufgibt, um zu promovieren. „An der Universität trifft Elinor ihren ,Seelenverwandten' Vincent Ostrom, einen Politikwissenschaftler. Zwischen den beiden entwickelt sich eine menschliche wie wissenschaftliche Symbiose“ (Horn 2009). Zusammen führen sie ein der Wissenschaft gewidmetes Leben. Glücklicherweise sind auch die Zeiten vorbei, in denen eine Frau aus Rücksicht auf die Karriere des Ehemannes nach den Regeln der Universität zurückstecken muss, wie es noch im Fall von Joan Violet Robinson zu erleben war. ${ }^{9}$

Die institutionenökonomische Ausrichtung beider Preisträger führt sie zusammen als Mitglieder der Institutional Society for the New Institutional Economics, dessen Präsident Oliver E. Ostrom 1999 bis 2001 war. Zu Deutschland - wie im Übrigen auch zu vielen anderen ausländischen Universitäten - hat Elinor Ostrom vielseitigen wissenschaftlichen Kontakt. Erst jüngst erhielt sie in Frankfurt den Reimer-Lüst-Preis. Dessen Preisgeld stiftet Elinor Ostrom dem von ihr initiierten deutsch-amerikanischen Austauschprogramm und ermöglicht so die Forschungskooperation mit der Humboldt-Universität Berlin sowie mit der Philipps-Universität Marburg. Dort arbeiten die auch in der Genossenschaftswissenschaft beheimateten Agrarökonomen Konrad Hagedorn und Michael Kirk, die schon seit einigen Jahren gemeinsam mit dem Kooperationswissenschaftler Markus Hanisch mit Elinor Ostrom regen wissenschaftlichen Austausch pflegen.

Die interdisziplinäre Ausrichtung beider Nobelpreisträger und ihre institutionenökonomische Ansätze führten dazu, dass beide als Hauptredner auf den letzten beiden Internationalen Genossenschaftswissenschaftlichen Tagungen (IGT) eingeladen waren: Oliver E. Williamson auf der von Theresia Theurl veranstalteten IGT 2004 in Münster und Elinor Ostrom auf der von Hans Jürgen Rösner mit Frank Schulz-Nieswandt organisierten IGT 2008 in Köln. Ebenfalls Gast auf der Kölner Veranstaltung war der mit dem Internationa-

9 So musste noch Joan Violet Robinson ihre Ehe mit dem Ökonomen Austin Robinson, aus der zwei Töchter hervorgingen, mit einem Karriereknick bezahlen, denn erst ,nach dem Ausscheiden ihres Mannes durfte sie Professorin in Cambridge werden. Ihre mit großer Begeisterung aufgenommene Antrittsvorlesung zum Thema „Neuer Merkantilismus“ hielt sie erst 1966 - mit dreiundsechzig Jahren“ (Hickel 1994 S. 177). 
len Wissenschaftspreis der Arbeitsgemeinschaft Genossenschaftswissenschaftlicher Institute (AGI) ausgezeichnete Friedensnobelpreisträger 2006, Muhammad Yunus, dessen Verdienste um die Gründung der Grameen Bank in Bangladesh und deren Mikrokreditvergabe an Arme gewürdigt wurden. Diese Beispiele machen Mut, denn sie zeigen, dass hybride Organisationen, Selbstorganisation, genossenschaftliche und genossenschaftsähnliche Selbsthilfeformen eine Forschungsarena bieten, die zu Nobelpreisen führen kann (Schmale 2009, S. 769).

\section{Literaturverzeichnis}

Coase, Ronald H. (1937), The Nature of the Firm, in: Economica, H. 4, S. 386-405.

Engelhardt, Werner Wilhelm (2001), "Die Verfassung der Allmende. Jenseits von Staat und Markt". Ein Kommentar zur deutschen Fassung des Werks von Elinor Ostrom, in: Zeitschrift für das gesamte Genossenschaftswesen (ZfgG), 51. Jg., S. 44-47.

Groth, Markus (2009), Transaktionskosten und die Gestaltung ökonomischer Austauschbeziehungen. Zum Nobelpreis an Oliver E. Williamson, in: Wirtschaftsdienst, Nr. 11, S. 770-776.

Hardin, Garrett (1968), The Tragedy of the Commons. Science 162, S. 1243-1248.

Hickel, Rudolf (1994), Genie im Männerzirkus. Joan Violet Robinson begründete den Linkskeynesianismus. Die Nonkonformistin dachte früh über den Umbau der Industriegesellschaft nach, in: Die großen Ökonomen. Leben und Werk der wirtschaftswissenschaftlichen Vordenker, hrsg. von Nikolaus Pieper, Stuttgart, S. 176.

Horn, Karen (2009), Ganz schön schlau. Die Politologin ist hoch renommiert - für etwas, was simpel erscheint: Nämlich, dass die Menschen selber am besten wissen, was gut für sie ist, in: Emma, Heft 5/2009, aus dem Internet abrufbar unter: http://www.emma.de/elinor_ostrom_2009_5.html (Zugriff: 15.02.2010).

Kliemt, Hartmut (2009), Wofür es 2009 den Nobelpreis gab, in: FAZ.NET, http://www.faz.net (Zugriff: 21.10.2009).

Kliemt, Hartmut (2009a), Zwischen Markt und Staat. Nobelpreis für Wirtschaftswissenschaften 2009 an Elinor Ostrom und Oliver E. Williamson, in: WiSt, H. 12, S. 643-646.

Nobelprize.org (2009), Nobel Prizes, http://nobelprize.org/nobel_prizes/economics/laureates/2009/index.html, (Zugriff: 10.02.2010).

Olson, Mancur (1998), Die Logik kollektiven Handelns. Kollektivgüter und die Theorie der Gruppen, 4. Aufl., Tübingen.

Ostrom, Elinor (1990), Governing the Commons: The Evolution of Institutions for Collective Action, New York. deutsch (1999): Die Verfassung der Allmende. Jenseits von Staat und Markt, Tübingen.

Ostrom, Elinor, Roy Gardener und James M. Walker (1994), Rules, Games and Common-Pool Resources, Ann Arbor.

Ostrom, Elinor (2007), “Große Vermögen sind in der Regel wirtschaftlich unproduktiv”. Die Politikwissenschaftlerin Elinor Ostrom über Ungleichheit, Sozialkapital und die überragende Bedeutung der Vielfalt, Interview mit Karen Horn, in: FAZ, 9. Mai, Nr. 107, S. 17.

Ostrom, Elinor (2005), Understanding Institutional Diversity, Princeton University Press.

Ostrom, Elinor (2009), Social Cooperation in Collective-Action Situation, in: Beiträge genossenschaftlicher Selbsthilfe zur wirtschaftlichen und sozialen Entwicklung, hrsg. von Rösner, Hans Jürgen und Frank SchulzNieswandt, Tagungsband der IGT 2008, Berlin, S. 49-69.

Richter, Rudolf (1990), Sichtweise und Fragestellungen der Neuen Institutionenökonomik, in: Zeitschrift für Wirtschafts- und Sozialwissenschaften, S. 571-591.

Schellschmidt, Henner (1997), Ökonomische Institutionenanalyse und Sozialpolitik. Gustav Schmoller und John R. Commons als Vertreter einer sozialreformerisch ausgerichteten Institutionenökonomik, Marburg.

Schmale, Ingrid (2009), Institutionelle Diversität ist wichtig. Zum Nobelpreis an Elinor Ostrom, in: Wirtschaftsdienst, Nr. 11, S. 765-769.

Universität Bielefeld (2010), Zentrum für interdisziplinäre Forschung, Publikationen, http://www.uni-bielefeld.de/ ZIF/Publikationen/nobelpreis_fuer_elinor_ostrom.html (Zugriff: 15.02.2010).

Voigt, Stefan (2002), Institutionenökonomik. Neue Ökonomische Bibliothek, UTB München.

Williamson, Oliver E. (1975), Markets and Hierarchies: Analysis and Antitrust Implications, New York.

Williamson, Oliver E. (1985), The Economic Institutions of Capitalism, New York; deutsch (1990): Die ökonomischen Institutionen des Kapitalismus, Tübingen.

Williamson, Oliver E. (2005), Networks - Organizational Solutions to Future Challenges, in: Economics of Interfirm Networks, hrsg. von Theresia Theurl, Tübingen, S. 3-27. 


\section{Ingrid Schmale}

Williamson, Oliver E. (2009), Transaction Cost Economics: The Natural progression, Nobel Prize Lecture; http:// nobelprize.org/nobel_prizes/economics/laureates/2009/williamson-lecture-slides.pdf (Zugriff: 15.02.2010).

Zerche, Jürgen, Ingrid Schmale und Johannes Blome-Drees (1998), Einführung in die Genossenschaftslehre. Genossenschaftstheorie und Genossenschaftsmanagement, München. 